Gazi University
Journal of Science
$\mathrm{http} / /$ dergipark.gov.tr/gujs

\title{
Estimation of Parameters of Topp-Leone Inverse Lomax Distribution in Presence of Right Censored Samples
}

\author{
Amal HASSAN ${ }^{1}$ (D), Doaa ISMAIL $^{2}$ \\ ${ }^{1}$ Department of Mathematical Statistics, Faculty of Graduate Studies for Statistical Research, Cairo University, Giza, Egypt \\ ${ }^{2}$ Modern Academy for Engineering and Technology, Maadi, Egypt \\ Highlights \\ - Topp-Leone inverse Lomax is proposed as a new model. \\ - Structure properties of the new model are provided. \\ - Reliability and parameter estimators are obtined from complete and Type II censored samples. \\ - Simulation as well as data analysis is worked out.
}

\section{Article Info}

Received:24 July 2020

Accepted:17 Jan 2021

\section{Keywords}

Inverse lomax

Topp-Leone family

Maximum likelihood

Censored samples

\begin{abstract}
In this paper, we deal with a three-parameter inverse Lomax cited as the Topp-Leone inverse Lomax (TLIL) distribution depend on Topp-Leone-G family. Expressions of its density and distribution functions are explored. The structure properties of suggested model are provided like quantile function, moments, incomplete moments and Rényi entropy. Maximum likelihood estimators of the TLIL distribution parameters along with reliability estimator are worked out via complete and type II censored samples. To investigate the statistical properties of estimates we present numerical illustration along with two real data.
\end{abstract}

\section{INTRODUCTION}

The inverse Lomax (IL) distribution is one of the notable lifetime models that gives an application in economics and actuarial sciences (see [1]). Kleiber [2] employed the IL to get Lorenz ordering relationship among ordered statistics. McKenzie et al. [3] applied the IL model on geophysical databases.

The IL distribution is a particular model from generalized beta distribution of the second kind. The IL distribution is the inverse of Lomax distribution. The probability density function (pdf) of the IL distribution with shape parameter $\gamma$ and scale parameter $\varphi$ is

$$
g(x ; \gamma, \varphi)=\gamma \varphi^{-\gamma} x^{\gamma-1}\left(1+\frac{x}{\varphi}\right)^{-(\gamma+1)} ; \quad x>0, \gamma, \varphi>0
$$

The associated cumulative distribution function (cdf) is

$$
G(x ; \gamma, \varphi)=\left(1+\frac{x}{\varphi}\right)^{-\gamma} ; x>0, \gamma, \varphi>0
$$


Rahman and Aslam [4] discussed Bayesian estimation of unknown shape parameter of IL model owing to various loss functions. Further, Bayesian prediction of future ordered observations from IL mixture model was discussed by Rahman and Aslam [5]. Singh et al. [6] discussed reliability estimator of the IL distribution in presence of type II censoring (TIIC). Jan and Ahmad [7] considered the behaving shape parameter of IL distribution through distinct approximation manner. Estimation of entropy for IL model was considered from multiple censored samples by Bantan et al. [8]. Recent extensions of the IL distribution for further usage have been provided by several researches, for instance the reader can refer to [9-13].

Recently, several researchers considered various generated families of continuous distributions in order to develop new models as well as provide great flexibility in modelling real data. Many families of distributions were submitted from dissimilar bounded or unbounded distributions. Two essential components, namely generator and a parent distribution, are desired to assign new families (see [14]). Jones [15] provided beta family owing to beta random variable. Alzaarteh et al. [16] proposed the widespread family using any non-negative continuous random variable $T$ as the generator, instead of the beta random variable. They defined this class as follows

$$
F(x)=\int_{0}^{W(G(\mathrm{x}))} r(t) d t,
$$

where $r(t)$ is the pdf of a non-negative continuous random variable $T$. This generated family is called "TX distribution". The transformation $W(G(x))$ verify that: $W(G(x)) \in[0, \infty)$ and it is a monotonic nondecreasing function. For a random variable $T$ has the Topp Leone (TL) distribution on $[0,1]$, the TL-G family has been proposed by AL-Shomrani et al. [17] with cdf given by

$$
F(x)=(1-(\bar{G}(x)))^{\alpha},
$$

where $\alpha$ is the shape parameter. The pdf corresponding to (3) is given by

$$
f(x)=2 \alpha g(x) \bar{G}(x)(1-(\bar{G}(x)))^{\alpha-1},
$$

where $G(x)$ is the baseline distribution function and $g(x)$ is the associated density. Further, the TL-G family has been discussed by Rezaei et al. [18].

In many life-testing experiments, censoring is essential according to save the total time on test and to reduce the cost associated with the experiment. Various censoring methods are at hand to experimenter such as type-I in which the test stops at a pre-fixed time, and TIIC in which test stops at predetermined number of failures.

In the present work, we define a new model as a modification of the IL distribution with three-parameter. We provide some comprehensive descriptions of its statistical properties. Then we estimate the reliability function and population parameters of the model using the maximum likelihood (ML) method when the available data are drawn from complete and TIIC. The layout of the paper consists of the following sections. In Section 2, we introduce the three-parameter TLIL distribution and the formation of the pdf and cdf of their expansions for stated distribution is given. Statistical properties of TLIL distribution are illustrated in Section 3. In Section 4, ML estimators, approximate confidence intervals (CIs) and reliability estimators are derived. Numerical study is given to assess the behaviour of estimates and analysis to real data is considered as appear in Section 5. Finally we come up with concluding remarks in Section 6. 


\section{TOPP-LEONE INVERSE LOMAX MODEL}

In this section, we define a three-parameter TLIL distribution. Expansions for the pdf and cdf, reliability, hazard and reversed hazard functions are also presented.

Inserting (2) in (3) yields TLIL distribution with cdf specified by

$$
F(x ; \alpha, \gamma, \varphi)=\left[1-\left\{1-\left(1+\frac{\varphi}{x}\right)^{-\gamma}\right\}^{2}\right]^{\alpha} ; \quad x, \alpha, \gamma, \varphi>0
$$

The associated pdf corresponding to (5) is given by

$$
f(x ; \alpha, \gamma, \varphi)=2 \alpha \gamma \varphi^{-\gamma} x^{\gamma-1}\left(1+\frac{x}{\varphi}\right)^{-(\gamma+1)}\left[1-\left(1+\frac{\varphi}{x}\right)^{-\gamma}\right]\left[1-\left\{1-\left(1+\frac{\varphi}{x}\right)^{-\gamma}\right\}^{2}\right]^{\alpha-1} ; x>0 ; \alpha, \gamma, \varphi>0 .
$$

Figure 1 shows possible shapes of TLIL pdf for certain values of parameters. The extra shape parameter $\alpha$ is considered as a manner to supply a more flexible TLIL distribution.

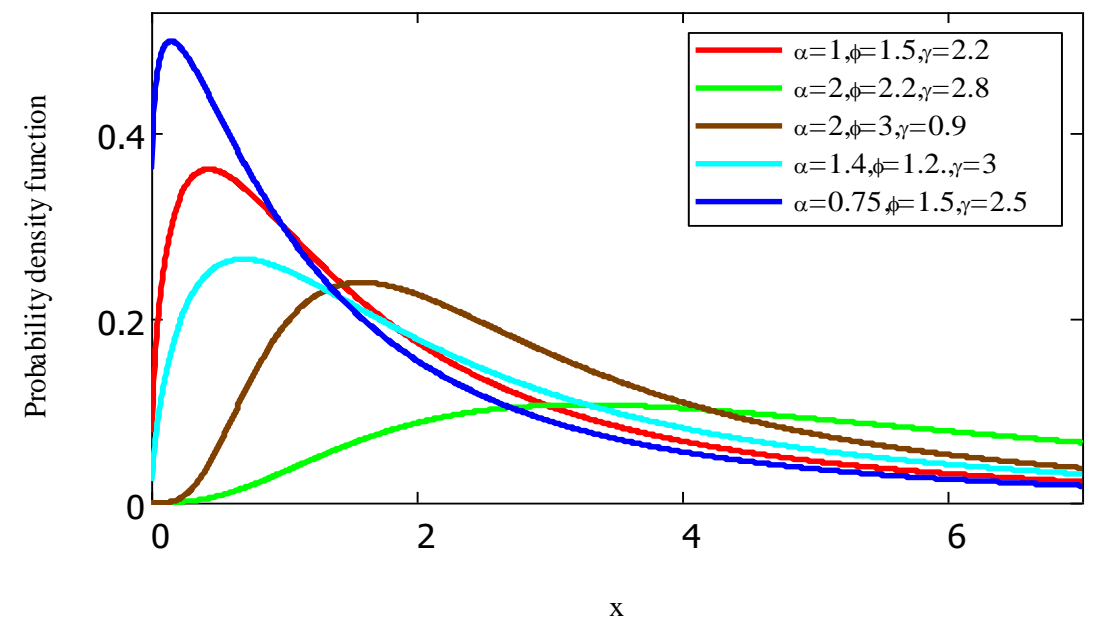

Figure 1. The pdf plots of TLIL distribution

\subsection{Expansions of pdf and cdf}

The binomial expansion, for real non-integer value of $\alpha$, is given by

$$
(1-D)^{\kappa}=\sum_{c=0}^{\infty} \frac{(-1)^{c} \Gamma(\kappa+1)}{c ! \Gamma(\kappa+1-j)} D^{c}
$$

Then, by applying the previous binomial series for pdf (6), so it can be formed as follows

$$
\left.f(x ; \alpha, \gamma, \varphi)=\sum_{j=0}^{\infty} \sum_{m=0}^{2 j+1} \eta_{j, m} \gamma \varphi^{-\gamma} x^{\gamma-1}\left(1+\frac{x}{\varphi}\right)^{-(\gamma+1)}\right)\left(1+\frac{\varphi}{x}\right)^{-\gamma m},
$$

where 
$\eta_{j, m}=\frac{(-1)^{j+m} 2 \Gamma(\alpha+1)}{j ! \Gamma(\alpha+1-j)(m+1)}\left(\begin{array}{l}2 j+1 \\ m\end{array}\right)$.

Also, it can be expressed as follows

$$
f(x ; \alpha, \gamma, \varphi)=\sum_{j=0}^{\infty} \sum_{m=0}^{2 j+1} w_{j, m} k_{m+1}(x),
$$

where $w_{j, m}=\frac{\eta_{j, m}}{(m+1)}$, and $k_{m+1}(x)=(m+1) g(x)(G(x))^{m}$.

Note that $k_{m+1}(x)$ is the exponentiated IL distribution with power parameter $m$. The expansion of the cdf is produced from expansion (7) for $s$ is a positive integer as follows

$$
[F(x ; \alpha, \gamma, \varphi)]^{s}=\sum_{i=0}^{\infty} \sum_{l=0}^{2 i} \zeta_{i, l}[G(x)]^{l}
$$

where $\zeta_{i, l}=\left(\begin{array}{c}2 i \\ l\end{array}\right) \frac{(-1)^{i+l} \Gamma(\alpha s+1)}{\Gamma(\alpha s+1-i) i !}$ and $G(x)$ is the cdf of IL distribution.

\subsection{Reliability Analysis}

The reliability function; $S(x ; \alpha, \gamma, \varphi)$, the hazard rate function (hrf); $\pi(x ; \alpha, \gamma, \varphi)$, cumulative hrf; $H(x ; \alpha, \gamma, \varphi)$, and reversed hrf;TLIL distribution are respectively given bythe of $R(x ; \alpha, \gamma, \varphi)$,

$$
\begin{aligned}
& S(x ; \alpha, \gamma, \varphi)=1-\left[1-\left\{1-(1+\varphi / x)^{-\gamma}\right\}^{2}\right]^{\alpha}, \\
& \pi(x ; \alpha, \gamma, \varphi)=\frac{2 \alpha \gamma \varphi^{-\gamma} x^{\gamma-1}\left(1+\frac{x}{\varphi}\right)^{-(\gamma+1)}\left[1-(1+\varphi / x)^{-\gamma}\right]\left[1-\left\{1-(1+\varphi / x)^{-\gamma}\right\}^{2}\right]^{\alpha-1}}{1-\left[1-\left\{1-(1+\varphi / x)^{-\gamma}\right\}^{2}\right]^{\alpha}} . \\
& H(x ; \alpha, \gamma, \varphi)=-\ln \left(1-\left[1-\left\{1-\left(1+\frac{\varphi}{x}\right)^{-\gamma}\right\}^{2}\right]^{\alpha}\right) .
\end{aligned}
$$

and,

$$
R(x ; \alpha, \gamma, \varphi)=2 \alpha \gamma \varphi^{-\gamma} x^{\gamma-1}\left[1-\left(1+\frac{\varphi}{x}\right)^{-\gamma}\right]\left(1+\frac{x}{\varphi}\right)^{-(\gamma+1)}\left[1-\left\{1-\left(1+\frac{\varphi}{x}\right)^{-\gamma}\right\}^{2}\right]^{-1} .
$$

Figure 2 shows a variety of possible shapes of hrf of TLIL distribution for specific values of parameters. 


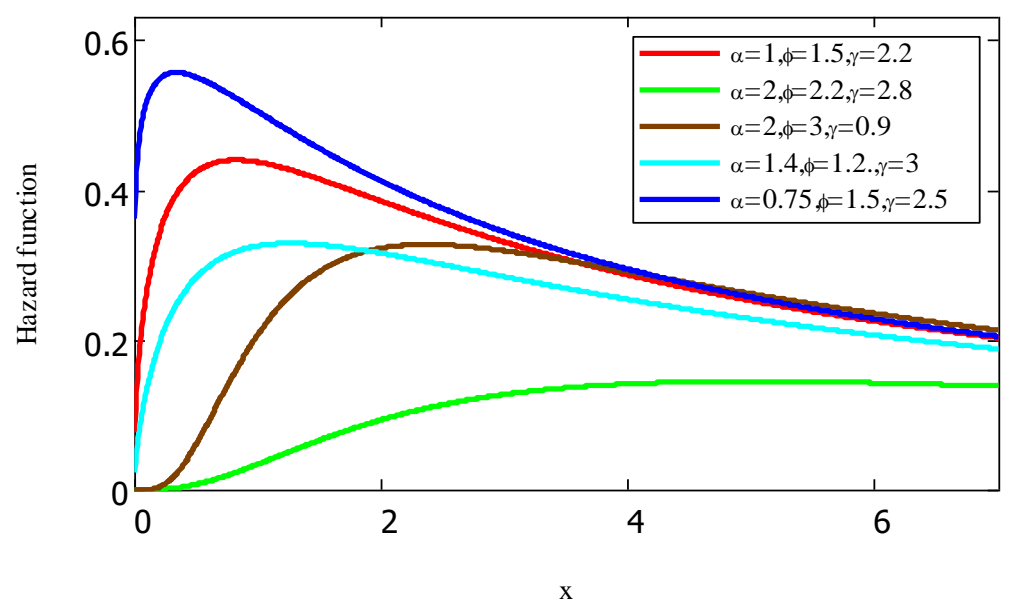

Figure 2. Plots of hrf of TLIL distribution

\section{PRINCIPLE PROPERTIES}

We derive some significant characteristics of the TLIL distribution, specifically; the $r^{\text {th }}$ moment, incomplete moments, moments of residual life function and Rényi entropy.

\subsection{Moments}

Moments in statistical analysis are important in study characteristics and shapes of distribution such that spread and dispersion which measured by mean and variance. It can study the flatness or peakedess of distribution which measured by kurtosis, also it can be used to study the symmetry of shape of distribution which measured by skewness. The $r^{\text {th }}$ moment for the TLIL can be obtained from pdf (8) as follows

$\mu_{r}^{\prime}=\sum_{j=0}^{\infty} \sum_{m=0}^{2 j+1} \eta_{j, m} \int_{0}^{\infty} \gamma \varphi^{-\gamma} x^{r+\gamma-1}\left(1+\frac{x}{\varphi}\right)^{-(\gamma+1)}\left(1+\frac{\varphi}{x}\right)^{-\gamma m} \mathrm{~d} x$

Hence the $r^{\text {th }}$ moment of TLIL is obtained as follows

$$
\mu_{r}^{\prime}=\sum_{j=0}^{\infty} \sum_{m=0}^{2 j+1}(-1)^{j+m} \eta_{j, m} \gamma \varphi^{r} \frac{\Gamma(1-r) \Gamma(r+\gamma+\gamma m)}{\Gamma(1+\gamma+\gamma m)}
$$

Fisher and K1lıcman [19] proved the following

$$
\Gamma(0)=-\varepsilon, \Gamma(-r)=\frac{(-1)^{r}}{r !} \delta(r)-\frac{(-1)^{r}}{r !} \varepsilon \text { for } r=1,2, \ldots
$$

where $\varepsilon$ denotes Euler's constant, and $\delta(r)=\sum_{i=1}^{r} \frac{1}{i}$.

Setting $r=1,2,3$ and 4 in (10), we can obtain the first four moments about zero. The mean and $\left(\mu_{1}^{\prime}\right)$ variance $\left(\sigma^{2}\right)$ of the TLIL distribution for some selected values of the parameters which can be calculated numerically in Table 1. 
Table 1. Mean and variance of TLIL distribution

\begin{tabular}{|l|l|l|l|l|l|l|l|}
\hline \multirow{2}{*}{$\alpha$} & \multirow{2}{*}{$\varphi$} & \multicolumn{2}{l|}{$\gamma=1$} & \multicolumn{2}{l|}{$\gamma=1.5$} & $\gamma=2$ & \\
\cline { 3 - 8 } & & $\mu_{1}^{\prime}$ & $\sigma^{2}$ & $\mu_{1}^{\prime}$ & $\sigma^{2}$ & $\mu_{1}^{\prime}$ & $\sigma^{2}$ \\
\hline \multirow{3}{*}{1.5} & 0.02 & 0.027 & 0.042 & 0.044 & 0.093 & 0.062 & 0.164 \\
\cline { 2 - 8 } & 0.5 & 0.687 & 26.14 & 1.106 & 58.175 & 1.541 & 102.59 \\
\cline { 2 - 8 } & 1 & 1.356 & 104.65 & 2.212 & 232.70 & 3.082 & 410.36 \\
\hline \multirow{4}{*}{2.5} & 0.02 & 0.039 & 0.069 & 0.062 & 0.154 & 0.086 & 0.272 \\
\cline { 2 - 8 } & 0.5 & 0.973 & 43.295 & 1.558 & 96.316 & 2.148 & 169.82 \\
\cline { 2 - 8 } & 1 & 1.945 & 173.18 & 3.115 & 385.26 & 4.295 & 679.27 \\
\hline \multirow{3}{*}{3.5} & 0.02 & 0.049 & 0.097 & 0.077 & 0.215 & 0.106 & 0.379 \\
\cline { 2 - 8 } & 0.5 & 1.218 & 60.347 & 1.931 & 134.23 & 2.647 & 236.64 \\
\cline { 2 - 7 } & 1 & 2.436 & 241.39 & 3.861 & 536.91 & 5.295 & 946.55 \\
\hline
\end{tabular}

Next, we derive a simple formula for the $r^{\text {th }}$ incomplete moment of $X$ defined by $\Lambda_{r}(y)=E\left(X^{r} \mid X<y\right)$. So, the quantity $\Lambda_{r}(y)$ comes from (8) as

$\Lambda_{r}(y)=\sum_{j=0}^{\infty} \sum_{m=0}^{2 j+1} \eta_{j, m} \gamma \varphi^{r} B\left(1-r, r+\gamma+\gamma m, \frac{\gamma}{\gamma+y}\right)$

where $B(.,, x)$ is the incomplete beta function. The incomplete moments are useful in fields like economics, reliability, demography, insurance and medicine.

\subsection{Skewness and Kurtosis}

The effect of each shape parameters $\alpha$ and $\gamma$ on the skewness and kurtosis of the TLIL distribution is considered here based on quantiles. The quantile function of the TLIL distribution say $Q(u)$ can be obtained as

$Q(u)=\varphi\left(\left[1-\sqrt{1-(u)^{\frac{1}{\alpha}}}\right]^{\frac{1}{-\gamma}}-1\right)^{-1}$,

where $u$ is a number between $(0,1)$. The Bowley' skewness and Moors' kurtosis based on Equation (11) are plotted for certain values of $\gamma$ as function of $\alpha$, and $\alpha$ as function of $\gamma$ (see Figures 3 and 4). These plots show that the skewness decreases when $\alpha$ gets larger for fixed $\varphi$ and when $\gamma$ increases for fixed $\varphi$. Figure 4 reveal that there is great flexibility of kurtosis curves. 

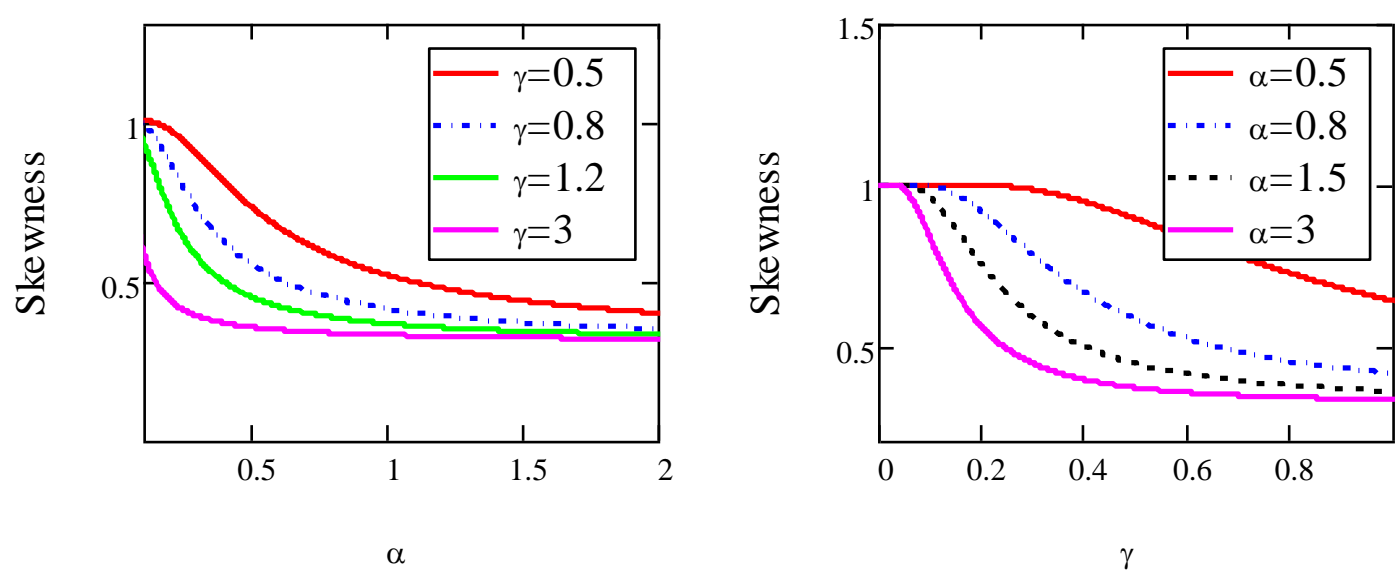

Figure 3. The Bowley' skewness of the TLIL distribution as a function of $\alpha$ and $\gamma$
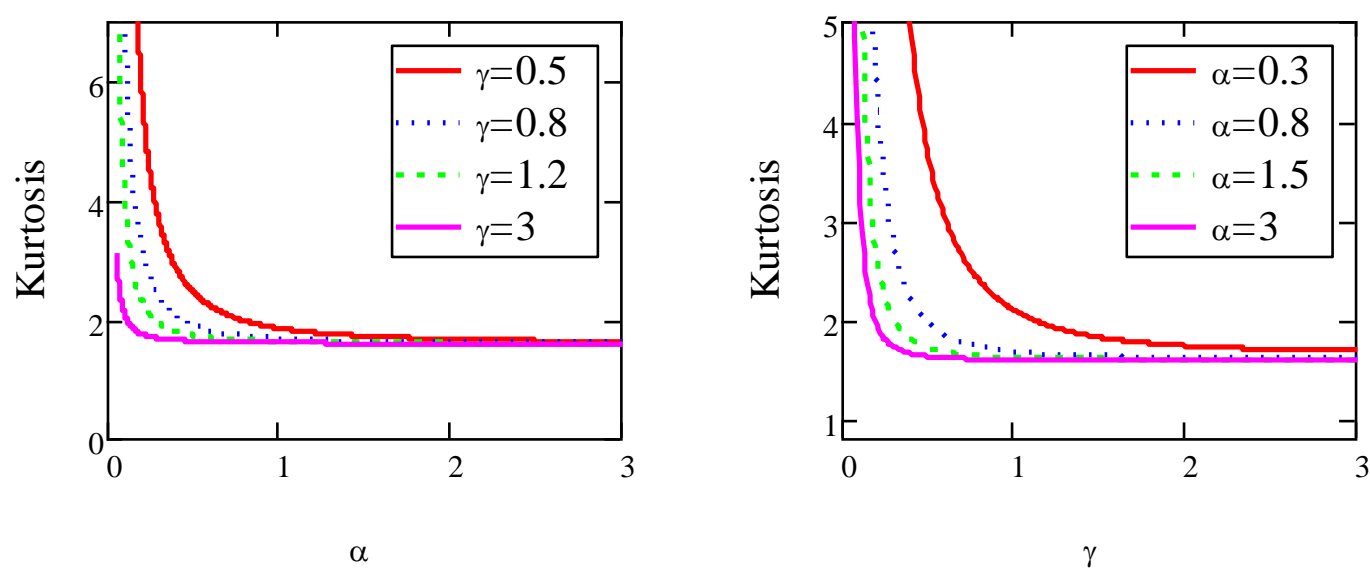

Figure 4. The Moors' kurtosis of the TLIL distribution as a function of $\alpha$ and $\gamma$

\subsection{Moments of Residual Life Function}

The $n^{\text {th }}$ moment of the residual life (RR) of TLIL model is given by

$m_{n}(y)=\frac{1}{S(y)} \int_{t}^{\infty}(x-y)^{n} f(x) d x$.

Hence, $n^{\text {th }}$ moment of RR of TLIL distribution is yielded by applying binomial expansion of $(x-y)^{n}$ as follows

$\left.m_{n}(y)=\frac{1}{S(y)} \sum_{j=0}^{\infty} \sum_{m=0}^{2 j+1} \sum_{k=0}^{n}(-1)^{n-k}\left(\begin{array}{l}n \\ k\end{array}\right) \gamma \varphi^{-\gamma} \eta_{j, m} \int_{y}^{\infty} x^{k} x^{\gamma-1} y^{n-k}\left(1+\frac{x}{\varphi}\right)^{-(\gamma+1)}\right)\left(1+\frac{\varphi}{x}\right)^{-\gamma m} d x$,

which leads to

$m_{n}(y)=\frac{1}{S(y)} \sum_{j=0}^{\infty} \sum_{m=0}^{2 j+1} \sum_{k=0}^{n}(-1)^{n-k}\left(\begin{array}{l}n \\ k\end{array}\right) y^{n-k} \gamma \varphi^{k} \eta_{j, m} \mathrm{~B}\left(1-k, k+\gamma+\gamma m, \frac{\varphi}{\varphi+y}\right)$, 
where $\mathrm{B}(., ., x)$ is the incomplete beta function. For $n=1$, we get the mean residual life of TLIL distribution which has many applications.

\subsection{Rényi Entropy}

The Rényi entropy of $X$ for continuous random variable with range $R$ is a measure of uncertainty. It is defined as follows

$$
v_{R}(X)=\frac{1}{1-\vartheta} \log \left\{\int_{R} f(x)^{\vartheta} d x\right\}, \quad \vartheta \neq 1, \vartheta>0
$$

To obtain Rényi entropy of the TLIL distribution, we must obtain explicit expression for $(f(x ; \alpha, \gamma, \varphi))^{\vartheta}$, as follows

$$
(f(x ; \alpha, \gamma, \varphi))^{\vartheta}=\sum_{j=0}^{\infty} \sum_{m=0}^{\infty} \frac{(-1)^{j+m}}{j !}\left(\begin{array}{c}
2 j+\vartheta \\
m
\end{array}\right) \frac{\left(2 \alpha \gamma \varphi^{-\gamma}\right)^{\vartheta} x^{\vartheta(\gamma-1)} \Gamma(\vartheta(\alpha-1)+1)}{\Gamma(\vartheta(\alpha-1)+1-j)}\left(1+\frac{\varphi}{x}\right)^{-\gamma m}\left(1+\frac{x}{\varphi}\right)^{-\vartheta(\gamma+1)} .
$$

Therefore, the Rényi entropy of TLIL distribution is given by

$$
v_{R}(x)=\frac{1}{1-\vartheta} \log \left\{\sum_{j=0}^{\infty} \sum_{m=0}^{\infty} L_{j, m} \frac{\Gamma(2 \vartheta-1) \Gamma(\vartheta(\gamma-1)+\gamma m+1)}{\Gamma(\vartheta m+\vartheta \gamma+\vartheta)}\right\}
$$

where

$$
L_{j, m}=\frac{(-1)^{j+m}}{j !}\left(\begin{array}{c}
2 j+\vartheta \\
m
\end{array}\right)(2 \alpha \gamma)^{\vartheta} \frac{\Gamma(\vartheta(\alpha-1)+1)}{\Gamma(\vartheta(\alpha-1)+1-j)} \varphi^{1-\vartheta}
$$

Furthermore, the $\varpi-$ entropy, where $\varpi>0, \varpi \neq 1$, is given by

$$
H_{\varpi}(x)=\frac{1}{\varpi-1}\left(1-\left\{\sum_{j=0}^{\infty} \sum_{m=0}^{\infty} L_{j, m} \frac{\Gamma(2 \varpi-1) \Gamma(\varpi(\gamma-1)+\gamma m+1)}{\Gamma(\varpi m+\varpi \gamma+\varpi)}\right\}\right) .
$$

\section{PARAMETER ESTIMATION}

This section deals with parameter and reliability function estimators for TLIL distribution from complete and TIIC samples. ML estimators are obtained as well as the approximate CIs are constructed.

The ТПС scheme is observed when $n$ units are placed on test, and the test is stopped at the time of the $h^{\text {th }}$ failure. It has the advantage that the number of observed failures is fixed to be $h$ which ensures reasonable information is available for statistical analysis.

Suppose that $n$ items in which their lifetimes follow TLIL distribution (6) are put on test and the test is stopped at fixed value of $h$ failure. The likelihood function for the observed samples $X_{(1)}<X_{(2)}<\ldots<X_{(h)}$ is given by

$$
L(\alpha, \gamma, \varphi ; \underline{x})=\frac{n !}{(n-h) !} \prod_{i=1}^{h} \frac{2 \alpha \gamma}{\varphi^{\gamma}} \frac{x_{(i)}^{\gamma-1}}{\left(1+\frac{x_{(i)}}{\varphi}\right)^{\gamma+1}}\left[1-\left(1+\frac{\varphi}{x_{(i)}}\right)^{-\gamma}\right]\left[1-\left\{1-\left(1+\frac{\varphi}{x_{(i)}}\right)^{-\gamma}\right\}^{2}\right]^{\alpha-1}\left(1-\left[1-\left\{1-\left(1+\frac{\varphi}{x_{(h)}}\right)^{-\gamma}\right\}^{2}\right]^{\alpha}\right)^{n-h} .
$$


For simplicity, write $x_{i}$ instead of $x_{(i)}$. The logarithm of the previous equation, denoted by, $\ln L$, takes the following form

$\ln L \propto h \ln \alpha+h \ln \gamma-h \gamma \ln \varphi+(\gamma-1) \sum_{i=1}^{h} \ln \left(x_{i}\right)-(\gamma+1) \sum_{i=1}^{h} \ln \left(1+\frac{x_{i}}{\varphi}\right)+\sum_{i=1}^{h} \ln z_{i}+(\alpha-1) \sum_{i=1}^{h} \ln \left(1-z_{i}{ }^{2}\right)+$ $(n-h) \ln \left(1-\left(1-z_{h}^{2}\right)^{\alpha}\right)$.

where $z_{i}=1-\left(1+\frac{\varphi}{x_{i}}\right)^{-\gamma}, z_{h}=1-\left(1+\frac{\varphi}{x_{h}}\right)^{-\gamma}$.

The components of the score vector $(\partial \ln L / \partial \varphi, \partial \ln L / \partial \alpha, \partial \ln L / \partial \gamma)$ are given below

$$
\begin{aligned}
\frac{\partial \ln L}{\partial \varphi} & =\frac{-h \gamma}{\varphi}+(\gamma+1) \sum_{i=1}^{h} \frac{x_{i}}{\varphi\left(\varphi+x_{i}\right)}+\sum_{i=1}^{h} \frac{1}{z_{i}} \frac{\partial z_{i}}{\partial \varphi}-2(\alpha-1) \sum_{i=1}^{h} \frac{z_{i}}{1-z_{i}{ }^{2}} \frac{\partial z_{i}}{\partial \varphi} \\
& +(n-h) \frac{2 \mathrm{z}_{h} \alpha\left(1-\mathrm{z}_{h}{ }^{2}\right)^{\alpha-1}}{1-\left(1-\mathrm{z}_{h}{ }^{2}\right)^{\alpha}} \frac{\partial z_{h}}{\partial \varphi}, \\
\frac{\partial \ln L}{\partial \alpha} & =\frac{h}{\alpha}+\sum_{i=1}^{h} \ln \left(1-z_{i}{ }^{2}\right)-(n-h) \frac{\left(1-\mathrm{z}_{h}{ }^{2}\right)^{\alpha} \ln \left(1-\mathrm{z}_{h}{ }^{2}\right)}{1-\left(1-\mathrm{z}_{h}{ }^{2}\right)^{\alpha}},
\end{aligned}
$$

and

$$
\begin{aligned}
\frac{\partial \ln L}{\partial \gamma} & =\frac{h}{\gamma}-h \ln \varphi+\sum_{i=1}^{h} \ln x_{i}-\sum_{i=1}^{h} \ln \left(1+\frac{x_{i}}{\varphi}\right)+(\alpha-1) \sum_{i=1}^{h} \frac{2 z_{i}}{1-z_{i}{ }^{2}} \frac{\partial z_{i}}{\partial \gamma}+\sum_{i=1}^{h} \frac{1}{z_{i}} \frac{\partial z_{i}}{\partial \gamma} \\
& +(n-h) \frac{2 \alpha \mathrm{z}_{h}\left(1-\mathrm{z}_{h}{ }^{2}\right)^{\alpha-1}}{1-\left(1-\mathrm{z}_{h}{ }^{2}\right)^{\alpha}} \frac{\partial z_{h}}{\partial \gamma} \\
\frac{\partial z_{i}}{\partial \varphi}= & \gamma\left(1+\frac{\varphi}{x_{i}}\right)^{-\gamma-1}\left(\frac{1}{x_{i}}\right), \frac{\partial z_{i}}{\partial \gamma}=\left(1+\frac{\varphi}{x_{i}}\right)^{-\gamma} \ln \left(1+\frac{\varphi}{x_{i}}\right) .
\end{aligned}
$$

The ML estimators of the model parameters are produced after solving the non-linear Equations (12) - (14) numerically. Also, the ML estimators in case of complete sample are obtained by setting $h=n$ in previous equations. However, it is difficult to find a closed form solution for the above equations; so an iterative procedure is applied to obtain ML estimates (MLEs).

Based on invariance property of ML estimation, we obtain the ML estimator of $S(x)$ by replacing the parameters from (12) - (14) by their ML estimators as follows:

$\hat{S}(x ; \hat{\alpha}, \hat{\gamma}, \hat{\varphi})=1-\left[1-\left\{1-(1+\hat{\varphi} / x)^{-\hat{\gamma}}\right\}^{2}\right]^{\hat{\alpha}}$.

For interval estimation of the parameters, the observed information matrix $I(\Psi)=\left\{I_{u v}\right\}$ for $(u, v)=(\alpha, \gamma, \varphi)$ must be obtained. Under the regularity conditions, asymptotic variance-covariance matrix of the MLEs of $\alpha, \varphi$ and $\gamma$ can obtained by inverting the following observed information matrix, 
$I^{-1}(\hat{\Psi})=\left[\begin{array}{ccc}\operatorname{var}(\hat{\alpha}) & \operatorname{cov}(\hat{\alpha}, \hat{\varphi}) & \operatorname{cov}(\hat{\alpha}, \hat{\gamma}) \\ \operatorname{cov}(\hat{\alpha}, \hat{\varphi}) & \operatorname{var}(\hat{\varphi}) & \operatorname{cov}(\hat{\varphi}, \hat{\gamma}) \\ \operatorname{cov}(\hat{\alpha}, \hat{\gamma}) & \operatorname{cov}(\hat{\varphi}, \hat{\gamma}) & \operatorname{var}(\hat{\gamma})\end{array}\right]=\frac{1}{|I|}\left[\begin{array}{ccc}\partial^{2} \ln L / \partial \alpha^{2} & \partial^{2} \ln L / \partial \alpha \partial \varphi & \partial^{2} \ln L / \partial \alpha \partial \gamma \\ \partial^{2} \ln L / \partial \alpha \partial \varphi & \partial^{2} \ln L / \partial \varphi^{2} & \partial^{2} \ln L / \partial \varphi \partial \gamma \\ \partial^{2} \ln L / \partial \alpha \partial \gamma & \partial^{2} \ln L / \partial \varphi \partial \gamma & \partial^{2} \ln L / \partial \gamma^{2}\end{array}\right]_{\alpha=\hat{\alpha}, \varphi=\hat{\varphi}, \gamma=\hat{\gamma}}$

The asymptotic normality of ML estimation can be used to compute the asymptotic $100(1-v) \%, 0<v<1$, confidence limits for $\alpha, \gamma$ and $\varphi$ as follows

$\hat{\alpha} \pm z_{v / 2} \sqrt{\operatorname{var}(\hat{\alpha})},\left(\hat{\gamma} \pm z_{v / 2} \sqrt{\operatorname{var}(\hat{\gamma})}\right.$, and $\left(\hat{\varphi} \pm z_{v / 2} \sqrt{\operatorname{var}(\hat{\varphi})}\right.$,

where $z_{v / 2}$, standard normal percentile and $(1-v)$ is the confidence coefficient. The asymptotic $100(1-v) \%$ confidence limits for reliability function is given by

$\hat{S}(x) \pm z_{v / 2} \sqrt{\operatorname{var}(\hat{S}(x))}$.

\section{SIMULATION STUDY}

We present numerical study to examine the behaviour of the ML and reliability function estimates. Measures like mean square errors (MSEs), relative bias (RB), standard errors (SEs), lower bound (LB) of CIs, upper bound (UB) of CIs, and average length (Le) of 95\% CIs are calculated. We perform the following algorithm.

* 1000 random sample of sizes 50, 200 and 300 are generated from the TLIL distribution.

* The number of failure items; $h$, is chosen as $90 \%$ (censoring scheme) and $100 \%$ (complete sample).

* Parameters values are specified as (i) $(\alpha=1, \gamma=0.8, \varphi=2)$, (ii) $(\alpha=1.25, \gamma=0.8, \varphi=2)$, (iii) ( $\alpha=0.8, \gamma=1.5, \varphi=2)$, (iv) ( $\alpha=1.3, \gamma=1, \varphi=1)$, (v) ( $\alpha=1, \gamma=1.5, \varphi=0.5)$, (vii) $(\alpha=1.6, \gamma=0.8, \varphi=2)$.

* The MSEs, RB, SEs for all samples sizes and for all selected set of parameters are computed.

* The LB, UB and Le atfor all samples sizes and for all selected sets of parameters are $v=0.05$ computed.

* Reliability estimates and the associated $95 \%$ CI for reliability function at different mission time $t_{0}$ where $t_{0}=0.1,0.3,0.5$ for different sample sizes are presented.

\subsection{Numerical Results}

The observed outcomes are reported in Tables 2 to 9 , we detect the following about the performance of estimates:

- SE of all estimates decreases as $n$ increases. Also, it has the smallest values in complete sample (see Tables 2 and 3).

- The MSEs and RBs of $\alpha, \gamma$ and $\varphi$ estimates decrease as $n$ increases for all cases. Also, the MSEs and RBs at $h=0.9 n$ for all estimates are greater than the corresponding at $h=n$ (see Tables 2 and $3)$.

- The MSEs and SEs of $\gamma$ estimates are less than the corresponding for $\alpha$ and $\varphi$ estimates in almost all cases (see Tables 2 and 3).

- For all estimates of parameters, as the value of failure items; $h$ increases the MSEs, RBs and SEs decrease.

- For fixed value of $\varphi=2$, sestimate $\alpha$ of sand RB sincreases, the MSE $\alpha=0.8$ and as the value of $\gamma$ decrease at the same sample size (see Table 2).

- For all cases, it is clear that the Le of CIs for MLEs decrease as $n$ increases (see Tables 4 and 5). 
- The MSE for $\alpha$ estimates gets the smallest values at $\alpha=1.3, \gamma=1$ and $\varphi=1$ compared to other cases. The MSE for $\varphi$ estimates at $\alpha=0.8, \gamma=1.5$ andhas the smallest value compared to $\varphi=2$ other cases. The MSE of $\gamma$ estimates at $\alpha=1, \gamma=0.8$ andables Thas the smallest value (see $\varphi=2$ 2 and 3$)$.

- For all cases, reliability estimates decrease as the mission time's increase. Also, the Le of CIs gets shorter as $n$ increases (see Tables 6-8).

Table 2. The MSEs RBs and SEs of the estimates for sets (i), (ii) and (iii)

\begin{tabular}{|c|c|c|c|c|c|c|c|c|c|c|c|}
\hline \multirow{2}{*}{$n$} & \multirow{2}{*}{$h$} & \multirow{2}{*}{$\begin{array}{l}\text { Criteria } \\
\text { Measures }\end{array}$} & \multicolumn{3}{|c|}{$\alpha=1, \gamma=0.8, \varphi=2$} & \multicolumn{3}{|c|}{$\alpha=1.25, \gamma=0.8, \varphi=2$} & \multicolumn{3}{|c|}{$\alpha=0.8, \gamma=1.5, \varphi=2$} \\
\hline & & & $\hat{\alpha}$ & $\hat{\gamma}$ & $\hat{\varphi}$ & $\hat{\alpha}$ & $\hat{\gamma}$ & $\hat{\varphi}$ & $\hat{\alpha}$ & $\hat{\gamma}$ & $\hat{\varphi}$ \\
\hline \multirow{6}{*}{50} & \multirow{3}{*}{45} & MSE & 0.241 & 0.005 & 0.247 & 0.113 & 0.010 & 0.184 & 0.560 & 0.303 & 0.552 \\
\hline & & $\mathrm{RB}$ & 0.453 & 0.038 & 0.089 & 0.184 & 0.048 & 0.043 & 0.855 & 0.349 & 0.273 \\
\hline & & SE & 0.004 & 0.001 & 0.009 & 0.005 & 0.002 & 0.008 & 0.006 & 0.003 & 0.010 \\
\hline & \multirow{3}{*}{50} & MSE & 0.231 & 0.005 & 0.202 & $\begin{array}{l}0.109 \\
\end{array}$ & 0.011 & 0.153 & 0.553 & 0.303 & 0.542 \\
\hline & & RB & 0.443 & 0.033 & 0.066 & 0.060 & 0.047 & 0.026 & 0.846 & 0.348 & 0.259 \\
\hline & & SE & 0.004 & 0.001 & 0.009 & 0.005 & 0.001 & 0.008 & 0.006 & 0.003 & 0.010 \\
\hline \multirow{6}{*}{200} & \multirow{3}{*}{180} & MSE & 0.036 & 0.004 & 0.109 & 0.024 & 0.010 & 0.095 & 0.331 & 0.278 & 0.397 \\
\hline & & RB & 0.151 & 0.007 & 0.035 & 0.034 & 0.078 & 0.007 & 0.686 & 0.345 & 0.248 \\
\hline & & SE & 0.001 & 0.000 & 0.002 & 0.001 & 0.000 & 0.002 & 0.001 & 0.001 & 0.002 \\
\hline & \multirow{3}{*}{200} & MSE & 0.031 & 0.004 & 0.109 & 0.022 & 0.009 & 0.094 & 0.327 & 0.276 & 0.396 \\
\hline & & RB & 0.152 & 0.001 & 0.033 & 0.033 & 0.074 & 0.004 & 0.682 & 0.344 & 0.226 \\
\hline & & SE & 0.001 & 0.000 & 0.002 & 0.001 & 0.000 & 0.002 & 0.001 & 0.001 & 0.002 \\
\hline \multirow{6}{*}{300} & \multirow{3}{*}{270} & MSE & 0.021 & 0.003 & 0.085 & 0.015 & 0.009 & 0.068 & 0.317 & 0.275 & 0.343 \\
\hline & & RB & 0.109 & 0.009 & 0.040 & 0.012 & 0.086 & 0.012 & 0.680 & 0.345 & 0.242 \\
\hline & & SE & 0.000 & 0.000 & 0.001 & 0.000 & 0.000 & 0.001 & 0.000 & 0.000 & 0.001 \\
\hline & \multirow{3}{*}{300} & MSE & 0.021 & 0.003 & 0.083 & 0.015 & 0.009 & 0.067 & 0.301 & 0.271 & 0.333 \\
\hline & & RB & 0.108 & 0.006 & 0.025 & 0.011 & 0.084 & 0.012 & 0.661 & 0.343 & 0.237 \\
\hline & & SE & 0.000 & 0.000 & 0.001 & 0.000 & 0.000 & 0.001 & 0.000 & 0.000 & 0.001 \\
\hline
\end{tabular}


Table 3. The MSEs, RBs and SEs of the estimates for sets (iv), (v) and (vi)

\begin{tabular}{|c|c|c|c|c|c|c|c|c|c|c|c|}
\hline \multirow{2}{*}{$n$} & \multirow{2}{*}{$h$} & \multirow{2}{*}{$\begin{array}{l}\text { Criteria } \\
\text { Measures }\end{array}$} & \multicolumn{3}{|c|}{$\alpha=1.3, \gamma=1, \varphi=1$} & \multicolumn{3}{|c|}{$\alpha=1, \gamma=1.5, \varphi=0.5$} & \multicolumn{3}{|c|}{$\alpha=1.6, \gamma=0.8, \varphi=2$} \\
\hline & & & $\hat{\alpha}$ & $\hat{\gamma}$ & $\hat{\varphi}$ & $\hat{\alpha}$ & $\hat{\gamma}$ & $\hat{\varphi}$ & $\hat{\alpha}$ & $\hat{\gamma}$ & $\hat{\varphi}$ \\
\hline \multirow{6}{*}{50} & \multirow{3}{*}{45} & MSE & 0.341 & 0.032 & 0.054 & 0.216 & 0.044 & 0.012 & 0.101 & 0.038 & 0.276 \\
\hline & & RB & 0.353 & 0.115 & 0.127 & 0.386 & 0.105 & 0.148 & 0.014 & 0.183 & 0.047 \\
\hline & & SE & 0.007 & 0.003 & 0.004 & 0.005 & 0.003 & 0.002 & 0.006 & 0.003 & 0.007 \\
\hline & \multirow{3}{*}{50} & MSE & 0.335 & 0.031 & 0.049 & 0.205 & 0.041 & 0.012 & 0.099 & 0.037 & 0.120 \\
\hline & & RB & 0.348 & 0.113 & 0.121 & 0.376 & 0.100 & 0.145 & 0.015 & 0.180 & 0.044 \\
\hline & & SE & 0.007 & 0.003 & 0.004 & 0.005 & 0.003 & 0.002 & 0.006 & 0.003 & 0.006 \\
\hline \multirow{6}{*}{200} & \multirow{3}{*}{180} & MSE & 0.114 & 0.014 & 0.029 & 0.085 & 0.048 & 0.008 & 0.052 & 0.033 & 0.092 \\
\hline & & $\mathrm{RB}$ & 0.209 & 0.082 & 0.066 & 0.385 & 0.048 & 0.270 & 0.088 & 0.196 & 0.058 \\
\hline & & $\mathrm{SE}$ & 0.001 & 0.000 & 0.001 & 0.001 & 0.001 & 0.000 & 0.001 & 0.000 & 0.001 \\
\hline & \multirow{3}{*}{200} & MSE & 0.111 & 0.014 & 0.029 & 0.077 & 0.041 & 0.008 & 0.049 & 0.029 & 0.091 \\
\hline & & RB & 0.204 & 0.080 & 0.066 & 0.225 & 0.105 & 0.084 & 0.079 & 0.183 & 0.057 \\
\hline & & SE & 0.001 & 0.000 & 0.001 & 0.001 & 0.001 & 0.000 & 0.001 & 0.000 & 0.001 \\
\hline \multirow{6}{*}{300} & \multirow{3}{*}{270} & MSE & 0.111 & 0.015 & 0.025 & 0.061 & 0.036 & 0.006 & 0.042 & 0.025 & 0.069 \\
\hline & & RB & 0.222 & 0.100 & 0.079 & 0.206 & 0.101 & 0.078 & 0.082 & 0.174 & 0.046 \\
\hline & & SE & 0.001 & 0.000 & 0.000 & 0.000 & 0.000 & 0.000 & 0.001 & 0.000 & 0.001 \\
\hline & \multirow{3}{*}{300} & MSE & 0.107 & 0.015 & 0.025 & 0.056 & 0.032 & 0.006 & 0.041 & 0.024 & 0.068 \\
\hline & & RB & 0.206 & 0.092 & 0.067 & 0.195 & 0.093 & 0.074 & 0.081 & 0.170 & 0.045 \\
\hline & & SE & 0.001 & 0.000 & 0.000 & 0.000 & 0.000 & 0.000 & 0.001 & 0.000 & 0.001 \\
\hline
\end{tabular}

Table 4. The LB, UB and Le of the estimates for sets (i), (ii) and (iii)

\begin{tabular}{|c|c|c|c|c|c|c|c|c|c|c|c|}
\hline & & & $\alpha=1$, & $=0.8,4$ & & $\alpha=1$ & $\gamma=0.8$ & $\rho=2$ & $\alpha=0$ & $\gamma=1.5$, & $=2$ \\
\hline$n$ & $h$ & & LB & UB & Le & LB & UB & Le & LB & $\mathrm{UB}$ & $\mathrm{Le}$ \\
\hline \multirow{6}{*}{50} & \multirow{3}{*}{45} & $\alpha$ & 1.080 & 1.524 & 0.444 & 0.999 & 1.599 & 0.600 & 0.889 & 1.665 & 0.775 \\
\hline & & $\gamma$ & 0.641 & 0.898 & 0.257 & 0.654 & 1.023 & 0.369 & 0.646 & 1.306 & 0.660 \\
\hline & & $\varphi$ & 1.267 & 3.087 & 1.820 & 1.262 & 2.909 & 1.647 & 1.501 & 3.531 & 2.029 \\
\hline & \multirow{3}{*}{50} & $\alpha$ & 1.092 & 1.532 & 0.439 & 1.005 & 1.599 & 0.594 & 0.889 & 1.640 & 0.751 \\
\hline & & $\gamma$ & 0.643 & 0.891 & 0.248 & 0.712 & 1.007 & 0.295 & 0.662 & 1.281 & 0.619 \\
\hline & & $\varphi$ & 1.384 & 3.099 & 1.715 & 1.395 & 2.590 & 1.195 & 1.536 & 3.557 & 2.021 \\
\hline \multirow{6}{*}{200} & \multirow{3}{*}{180} & $\alpha$ & 0.917 & 1.166 & 0.249 & 1.009 & 1.333 & 0.324 & 1.010 & 1.408 & 0.398 \\
\hline & & $\gamma$ & 0.687 & 0.925 & 0.238 & 0.716 & 1.009 & 0.293 & 0.781 & 1.184 & 0.403 \\
\hline & & $\varphi$ & 1.438 & 2.703 & 1.265 & 1.381 & 2.591 & 1.209 & 1.738 & 3.255 & 1.518 \\
\hline & \multirow{3}{*}{200} & $\alpha$ & 0.927 & 1.177 & 0.250 & 1.009 & 1.331 & 0.322 & 1.009 & 1.403 & 0.394 \\
\hline & & $\gamma$ & 0.683 & 0.915 & 0.232 & 0.717 & 1.011 & 0.294 & 0.783 & 1.186 & 0.403 \\
\hline & & $\varphi$ & 1.451 & 2.707 & 1.256 & 1.385 & 2.585 & 1.201 & 1.740 & 3.294 & 1.514 \\
\hline \multirow{6}{*}{300} & \multirow{3}{*}{270} & $\alpha$ & 0.942 & 1.155 & 0.213 & 1.028 & 1.294 & 0.266 & 1.058 & 1.385 & 0.327 \\
\hline & & $\gamma$ & 0.688 & 0.898 & 0.210 & 0.743 & 0.994 & 0.251 & 0.816 & 1.149 & 0.334 \\
\hline & & $\varphi$ & 1.532 & 2.630 & 1.098 & 1.466 & 2.485 & 1.019 & 1.837 & 3.131 & 1.294 \\
\hline & \multirow{3}{*}{300} & $\alpha$ & 0.941 & 1.151 & 0.209 & 1.034 & 1.297 & 0.264 & 1.048 & 1.369 & 0.322 \\
\hline & & $\gamma$ & 0.692 & 0.899 & 0.206 & 0.740 & 0.994 & 0.253 & 0.811 & 1.145 & 0.334 \\
\hline & & $\varphi$ & 1.541 & 2.625 & 1.085 & 1.461 & 2.477 & 1.016 & 1.846 & 3.136 & 1.290 \\
\hline
\end{tabular}


Table 5. The LB, UB and Le of the estimates for sets (iv), (v) and (vi)

\begin{tabular}{|c|c|c|c|c|c|c|c|c|c|c|c|}
\hline & & & $\alpha=$ & $3, \gamma=1$ & $\rho=1$ & $\alpha=$ & $\gamma=1.5$, & $=0.5$ & & $.6, \gamma=0$ & $\varphi=2$ \\
\hline$n$ & $h$ & & LB & UB & Le & LB & UB & Le & LB & UB & $\mathrm{Le}$ \\
\hline \multirow{6}{*}{50} & \multirow{3}{*}{45} & $\alpha$ & 1.050 & 2.014 & 0.964 & 0.879 & 1.518 & 0.638 & 1.008 & 1.814 & 0.805 \\
\hline & & $\gamma$ & 0.617 & 1.154 & 0.537 & 1.072 & 1.613 & 0.540 & 0.690 & 1.202 & 0.512 \\
\hline & & $\varphi$ & 0.751 & 1.484 & 0.733 & 0.411 & 0.737 & 0.327 & 1.261 & 2.553 & 1.292 \\
\hline & \multirow{3}{*}{50} & $\alpha$ & 1.031 & 1.994 & 0.964 & 0.883 & 1.500 & 0.681 & 1.010 & 1.816 & 0.806 \\
\hline & & $\gamma$ & 0.623 & 1.151 & 0.528 & 1.084 & 1.616 & 0.532 & 0.692 & 1.196 & 0.504 \\
\hline & & $\varphi$ & 0.756 & 1.487 & 0.730 & 0.408 & 0.736 & 0.328 & 0.937 & 2.201 & 1.263 \\
\hline \multirow{6}{*}{200} & \multirow{3}{*}{180} & $\alpha$ & 1.214 & 1.696 & 0.481 & 0.907 & 1.294 & 0.387 & 1.105 & 1.524 & 0.419 \\
\hline & & $\gamma$ & 0.731 & 1.067 & 0.335 & 1.067 & 1.583 & 0.517 & 0.782 & 1.132 & 0.350 \\
\hline & & $\varphi$ & 0.761 & 1.384 & 0.623 & 0.390 & 0.699 & 0.309 & 1.335 & 2.435 & 1.100 \\
\hline & \multirow{3}{*}{200} & $\alpha$ & 1.173 & 1.644 & 0.472 & 0.906 & 1.277 & 0.371 & 1.102 & 1.517 & 0.415 \\
\hline & & $\gamma$ & 0.751 & 1.088 & 0.337 & 1.092 & 1.592 & 0.501 & 0.786 & 1.135 & 0.349 \\
\hline & & $\varphi$ & 0.759 & 1.373 & 0.615 & 0.389 & 0.695 & 0.305 & 1.339 & 2.433 & 1.094 \\
\hline \multirow{6}{*}{300} & \multirow{3}{*}{270} & $\alpha$ & 1.263 & 1.643 & 0.380 & 0.939 & 1.243 & 0.303 & 1.163 & 1.516 & 0.353 \\
\hline & & $\gamma$ & 0.768 & 1.050 & 0.282 & 1.127 & 1.569 & 0.442 & 0.794 & 1.084 & 0.290 \\
\hline & & $\varphi$ & 0.809 & 1.348 & 0.538 & 0.405 & 0.672 & 0.267 & 1.427 & 2.388 & 0.961 \\
\hline & \multirow{3}{*}{300} & $\alpha$ & 1.246 & 1.621 & 0.376 & 0.933 & 1.231 & 0.298 & 1.165 & 1.518 & 0.353 \\
\hline & & $\gamma$ & 0.762 & 1.041 & 0.279 & 1.140 & 1.582 & 0.442 & 0.791 & 1.082 & 0.291 \\
\hline & & $\varphi$ & 0.809 & 1.345 & 0.537 & 0.405 & 0.669 & 0.264 & 1.429 & 2.390 & 0.961 \\
\hline
\end{tabular}

Table 6. MLEs and 95\% CI of reliability estimates for sets (i) and (ii) at $h=0.9$

\begin{tabular}{|c|c|c|c|c|c|c|c|c|c|c|}
\hline \multirow{3}{*}{$n$} & \multirow{3}{*}{$h$} & \multirow{3}{*}{$t_{0}$} & \multicolumn{4}{|c|}{$\alpha=1, \gamma=0.8, \varphi=2$} & \multicolumn{4}{|c|}{$\alpha=1.25, \gamma=0.8, \varphi=2$} \\
\hline & & & \multirow[t]{2}{*}{$\hat{S}$} & \multicolumn{3}{|l|}{ CI } & \multirow[t]{2}{*}{$\hat{S}$} & \multicolumn{3}{|l|}{ CI } \\
\hline & & & & LB & UB & $\mathrm{Le}$ & & LB & UB & $\mathrm{Le}$ \\
\hline \multirow[t]{3}{*}{50} & \multirow[t]{3}{*}{45} & 0.1 & 0.836 & 0.824 & 0.849 & 0.024 & 0.895 & 0.885 & 0.906 & 0.020 \\
\hline & & 0.3 & 0.645 & 0.630 & 0.661 & 0.031 & 0.730 & 0.715 & 0.744 & 0.029 \\
\hline & & 0.5 & 0.522 & 0.506 & 0.538 & 0.032 & 0.606 & 0.590 & 0.622 & 0.032 \\
\hline \multirow[t]{3}{*}{200} & \multirow[t]{3}{*}{180} & 0.1 & 0.833 & 0.830 & 0.836 & 0.006 & 0.894 & 0.892 & 0.897 & 0.004 \\
\hline & & 0.3 & 0.646 & 0.642 & 0.649 & 0.007 & 0.728 & 0.724 & 0.733 & 0.009 \\
\hline & & 0.5 & 0.523 & 0.519 & 0.527 & 0.007 & 0.605 & 0.601 & 0.609 & 0.007 \\
\hline \multirow[t]{3}{*}{300} & \multirow[t]{3}{*}{270} & 0.1 & 0.833 & 0.831 & 0.835 & 0.003 & 0.894 & 0.891 & 0.895 & 0.003 \\
\hline & & 0.3 & 0.647 & 0.644 & 0.649 & 0.004 & 0.727 & 0.725 & 0.730 & 0.004 \\
\hline & & 0.5 & 0.525 & 0.522 & 0.527 & 0.005 & 0.605 & 0.601 & 0.608 & 0.006 \\
\hline
\end{tabular}


Table 7. MLEs and 95\% CI of reliability estimates for sets (iii) and (iv) at $h=0.9$

\begin{tabular}{|c|c|c|c|c|c|c|c|c|c|c|}
\hline \multirow{3}{*}{$n$} & \multirow{3}{*}{$h$} & \multirow{3}{*}{$t_{0}$} & \multicolumn{4}{|c|}{$\alpha=0.8, \gamma=1.5, \varphi=2$} & \multicolumn{4}{|c|}{$\alpha=1.3, \gamma=1, \varphi=1$} \\
\hline & & & \multirow[t]{2}{*}{$\hat{S}$} & \multicolumn{3}{|l|}{ CI } & \multirow[t]{2}{*}{$\hat{\hat{S}}$} & \multicolumn{3}{|l|}{$\overline{\mathrm{CI}}$} \\
\hline & & & & LB & UB & $\mathrm{Le}$ & & $\overline{\mathrm{LB}}$ & UB & Le \\
\hline \multirow[t]{3}{*}{50} & \multirow[t]{3}{*}{45} & 0.1 & 0.957 & 0.951 & 0.963 & 0.012 & 0.899 & 0.889 & 0.909 & 0.019 \\
\hline & & 0.3 & 0.856 & 0.845 & 0.867 & 0.022 & 0.686 & 0.671 & 0.701 & 0.030 \\
\hline & & 0.5 & 0.761 & 0.748 & 0.774 & 0.026 & 0.530 & 0.513 & 0.546 & 0.034 \\
\hline \multirow[t]{3}{*}{200} & \multirow[t]{3}{*}{180} & 0.1 & 0.955 & 0.954 & 0.957 & 0.003 & 0.898 & 0.896 & 0.900 & 0.004 \\
\hline & & 0.3 & 0.853 & 0.850 & 0.855 & 0.005 & 0.688 & 0.684 & 0.691 & 0.007 \\
\hline & & 0.5 & 0.757 & 0.754 & 0.761 & 0.006 & 0.534 & 0.530 & 0.538 & 0.008 \\
\hline \multirow[t]{3}{*}{300} & \multirow[t]{3}{*}{270} & 0.1 & 0.956 & 0.955 & 0.957 & 0.002 & 0.897 & 0.895 & 0.899 & 0.003 \\
\hline & & 0.3 & 0.852 & 0.850 & 0.854 & 0.003 & 0.688 & 0.685 & 0.690 & 0.004 \\
\hline & & 0.5 & 0.757 & 0.755 & 0.759 & 0.004 & 0.534 & 0.531 & 0.536 & 0.005 \\
\hline
\end{tabular}

Table 8. MLEs and 95\% CI of reliability estimates for sets $v$ and vii at $h=0.9$

\begin{tabular}{|c|c|c|c|c|c|c|c|c|c|c|}
\hline \multirow{3}{*}{$n$} & & & \multicolumn{4}{|c|}{$\alpha=1, \gamma=1.5, \varphi=0.5$} & \multicolumn{4}{|c|}{$\alpha=1.6, \gamma=0.8, \varphi=2$} \\
\hline & \multirow[t]{2}{*}{$h$} & \multirow[t]{2}{*}{$t_{0}$} & \multirow{2}{*}{$\hat{S}$} & \multicolumn{3}{|l|}{$\mathrm{CI}$} & \multirow[t]{2}{*}{$\hat{\mathrm{S}}$} & \multicolumn{3}{|l|}{ CI } \\
\hline & & & & LB & UB & Le & & LB & UB & Le \\
\hline \multirow[t]{3}{*}{50} & \multirow[t]{3}{*}{45} & 0.1 & 0.871 & 0.860 & 0.882 & 0.022 & 0.944 & 0.937 & 0.952 & 0.015 \\
\hline & & 0.3 & 0.592 & 0.576 & 0.608 & 0.032 & 0.812 & 0.799 & 0.824 & 0.025 \\
\hline & & 0.5 & 0.415 & 0.399 & 0.432 & 0.033 & 0.695 & 0.680 & \begin{tabular}{|l|}
0.709 \\
\end{tabular} & 0.029 \\
\hline \multirow[t]{3}{*}{200} & \multirow[t]{3}{*}{180} & 0.1 & 0.869 & 0.866 & 0.872 & 0.005 & 0.943 & 0.941 & 0.945 & 0.003 \\
\hline & & 0.3 & 0.593 & 0.589 & 0.596 & 0.007 & 0.812 & 0.809 & 0.815 & 0.006 \\
\hline & & 0.5 & 0.416 & 0.412 & 0.420 & 0.008 & 0.696 & 0.693 & 0.700 & 0.007 \\
\hline \multirow[t]{3}{*}{300} & \multirow[t]{3}{*}{270} & 0.1 & 0.869 & 0.867 & 0.871 & 0.003 & 0.943 & 0.942 & 0.944 & 0.002 \\
\hline & & 0.3 & 0.593 & 0.591 & 0.596 & 0.005 & 0.811 & 0.809 & 0.813 & 0.004 \\
\hline & & 0.5 & 0.418 & 0.415 & 0.421 & 0.005 & 0.696 & 0.693 & 0.698 & 0.004 \\
\hline
\end{tabular}

\subsection{Data Analysis}

Two real data are provided to illustrate the importance of the TLIL distribution compared with IL, TL generalized exponential (TLGE) and TL inverse Weibull (TLIW). Kolmogorov- Smirnov goodness of fit test is obtained for each data set and the p-values in each distribution indicate that the model fits the data very well.

The first data set about time between failures for repairable item (see [20]). The second data reported by Jorgensen [21] represent active repair times (in hours) for airborne communication transceiver. To compare the fitted models, measures include; -2log-likelihood function evaluated at the parameter estimates, Akaike information criterion (AIC), Bayesian information criterion (BIC), consistent AIC (CAIC) and HannanQuinn information criterion (HQIC) are considered. The better model is corresponding to the smallest values of mentioned measures. Table 9 gives the results of selected measures.

Table 9. The statistics -2logL, AIC, CAIC, BIC and HQIC for the two real data sets

\begin{tabular}{|l|l|l|l|l|l|l|l|l|l|l|}
\hline \multicolumn{1}{|l|}{ Data I } & \multicolumn{2}{l|}{ Data $\Pi$} \\
\hline model & -2 LogL & AIC & BIC & CAIC & HQIC & -2 LogL & AIC & BIC & CAIC & HQIC \\
\hline TLIL & 99.63 & 105.63 & 109.84 & 106.84 & 106.98 & 187.78 & 193.76 & 198.85 & 194.45 & 99.63 \\
\hline TLGE & 600.25 & 606.25 & 610.46 & 607.46 & 607.60 & 195.39 & 201.39 & 206.45 & 202.05 & 600.25 \\
\hline TLIW & 325.54 & 331.54 & 335.74 & 332.46 & 332.88 & 224.82 & 230.82 & 235.89 & 231.49 & 325.54 \\
\hline IL & 199.01 & 205.01 & 209.22 & 205.94 & 206.36 & 314.48 & 320.48 & 325.55 & 321.15 & 199.01 \\
\hline
\end{tabular}


Additionally, MLEs, reliability estimates of TLIL distribution and their SEs for both real data based on TIIC are listed in Table 10.

Table 10. MLE, reliability estimate and SEs of TLIL distribution based on TIIC for both data

\begin{tabular}{|c|c|c|c|c|c|}
\hline $\begin{array}{l}\text { Real } \\
\text { data }\end{array}$ & $n$ & $h$ & estimator & Estimate & SEs \\
\hline I & 30 & 21 & $\begin{array}{l}\hat{\alpha} \\
\hat{\lambda} \\
\hat{\beta} \\
\hat{S}(0.1)\end{array}$ & $\begin{array}{l}0.502 \\
7.768 \\
0.369 \\
0.997\end{array}$ & $\begin{array}{l}0.016 \\
0.307 \\
0.010 \\
0.000\end{array}$ \\
\hline$\Pi$ & 40 & 28 & $\begin{array}{l}\hat{\alpha} \\
\hat{\lambda} \\
\hat{\beta} \\
\hat{S}(0.1)\end{array}$ & $\begin{array}{l}0.445 \\
34.602 \\
0.126 \\
1\end{array}$ & $\begin{array}{l}0.006 \\
0.556 \\
0.001 \\
0.000\end{array}$ \\
\hline
\end{tabular}

\section{CONCLUDING REMARKS}

In this paper, we introduce a three-parameter new model, so called, Topp-Leone inverse Lomax distribution. Explicit expressions for its density and distribution functions are proposed. Some of its statistical properties are derived. The ML estimators of population parameters are obtained as well as reliability estimator is derived in presence of complete and censored samples. The approximate confidence intervals of parameters together with interval of reliability estimator are provided. Simulation study is implemented to check the behaviour of proposed estimators and recommendations are reported based on simulation outcomes. The applicability and importance of the new model is proved empirically using two real data sets. Indeed, the TLIL model provides a better fit to these data than some other models.

\section{ACKNOWLEDGMENTS}

The authors are grateful to the editor and referees for their useful comments and suggestions for improvement which undoubtedly enhanced the presentation and quality of the article.

\section{CONFLICTS OF INTEREST}

No conflict of interest was declared by the authors.

\section{REFERENCES}

[1] Kleiber, C., Kotz, S., "Statistical size distributions in economics and actuarial sciences", Wiley, New York, (2003).

[2] Kleiber, C., "Lorenz ordering of order statistics from log-logistic and related distributions", Journal of Statistical Planning and Inference, 120: 13-19, (2004).

[3] McKenzie, D., Miller, C., Falk, D. A., "The Landscape ecology of fire”, Springer, New York, (2011).

[4] Rahman, J. Aslam, M., Ali, S., "Estimation and prediction of inverse Lomax model via Bayesian approach", Caspian Journal of Applied Science Research, 3: 43-56, (2013).

[5] Rahman, J., Aslam, M., "Interval prediction of future order statistics in two component mixture inverse Lomax model: A Bayesian approach", American Journal of Mathematical and Management Science, 33(3): 216-227, (2014). 
[6] Singh, S. K., Singh, U., Yadav, A. S., "Reliability estimation for inverse Lomax distribution under type $\Pi$ censored data using Markov chain Monte Carlo method", International Journal of Mathematics and Statistics, 17(1): 128-146, (2016).

[7] Jan, U. Ahmad, S.P., "Bayesian analysis of inverse Lomax distribution using approximation techniques", Mathematical Theory and Modeling, 7(7): 1-12, (2017).

[8] Bantan, R. A. R., Elgarhy, M., Chesneau, C., Jamal, F., "Estimation of entropy for inverse Lomax distribution under multiple censored data", Entropy, 22(601): 1-15, (2020).

[9] Hassan, A.S., Abd-Allah, M., "On the inverse power Lomax distribution”, Annals of Data Science, 6: 259-278, (2018).

[10] Hassan, A.S., Mohamed, R.E., "Weibull inverse Lomax distribution", Pakistan Journal of Statistics and Operation Research", 15: 587-603, (2019).

[11] Hassan, A.S., Mohamed, R.E., "Parameter estimation for inverted exponentiated Lomax distribution with right censored data", Gazi University Journal of Science, 32(4): 1370-1386, (2019).

[12] ZeinEldin, R.A., Haq, M.A.U., Hashmi, S., Elsehety, M., "Alpha power transformed inverse Lomax distribution with different methods of estimation and applications", Complexity, 1-15, (2020).

[13] Maxwell, O., Chukwu, A.U., Oyamakin, O.S., Khaleel, M.A., "The Marshall-Olkin inverse Lomax distribution (MO-ILD) with application on cancer stem cell", Journal of Advances in Mathematics and Computer Science, 33: 1-12, (2019).

[14] Jones, M. C., "Families of distributions arising from distributions of order statistics", TEST, 13 (1): $1-43,(2004)$.

[15] Eugene, N., Lee C., Famoye, F., "Beta-normal distribution and its applications", Communications in Statistics -Theory and Methods, 31: 497-512, (2002).

[16] Alzaarteh, A., Lee, C., Famoye, F., "A new method for generating families of continuous distribution", Merton, 71: 63-79, (2013).

[17] AL-Shomrani, A., Arif, O., Shawky, A., Hanif, S., Shahbaz, M., Q., "Topp-Leone family of distributions: Some properties and applications", Pakistan Journal of Statistics and Operation Research, 12(3): 443-451, (2016).

[18] Rezaei, S., Sadr, B. B., Alizadeh, M., Nadarajah, S., "Topp-Leone generated family of distributions: Properties and applications", Communication in Statistics-Theory and Methods, 46(6): 2893-2909, (2017).

[19] Fisher, B., K1licman, A., "Some results on the gamma function for negative integers", Applied Mathematics \& Information Sciences, 6: 173-176, (2012).

[20] Murthy, D. N. P., Xie, M., Jiang, R., Weibull models, 1st edition, John Wiley, New Jersey, (2004).

[21] Jorgensen, B., "Statistical Properties of the Generalized Inverse Gaussian Distribution", SpringerVerlag, New York, (1982). 